\title{
miR-155 Modifies Inflammation, Endothelial Activation and Blood-Brain Barrier Dysfunction in Cerebral Malaria
}

\author{
Kevin R Barker, ${ }^{1,2}$ Ziyue Lu, ${ }^{2}$ Hani Kim, ${ }^{2 *}$ Ying Zheng, ${ }^{3}$ Junmei Chen, ${ }^{4}$ Andrea L Conroy, ${ }^{2 * *}$ \\ Michael Hawkes, ${ }^{5}$ Henry S Cheng, ${ }^{1,6}$ Makon-Sébastien Njock, ${ }^{6 * *}$ Jason E Fish, ${ }^{1,6}$ John M Harlan, ${ }^{7}$ \\ Jose A López, ${ }^{4,7}$ W Conrad Liles, ${ }^{7+}$ and Kevin C Kain ${ }^{1,2+}$
}

${ }^{1}$ Department of Laboratory Medicine and Pathobiology, University of Toronto, Ontario, Canada; ${ }^{2}$ Sandra Rotman Centre for Global Health, University Health Network-Toronto General Hospital, and Tropical Disease Unit, Department of Medicine, University of Toronto, Ontario, Canada, and *current affiliation: Bill and Melinda Gates Foundation, Seattle, Washington, United States of America; **Department of Pediatrics, Indiana University, Indianapolis, Indiana, United States of America; ${ }^{3}$ Department of Bioengineering and Center of Cardiovascular Biology, Institute of Stem Cell and Regenerative Medicine, University of Washington, Seattle, Washington, United States of America; ${ }^{4}$ Bloodworks Northwest Research Institute, Seattle, Washington, United States of America; ${ }^{5}$ Division of Infectious Diseases, Department of Pediatrics, University of Alberta, Edmonton, Alberta, Canada; ${ }^{6}$ Toronto General Research Institute, University Health Network, and Heart and Stroke Richard Lewar Centre of Excellence in Cardiovascular Research, Toronto, Ontario, Canada, and *** current affiliation: Laboratory of Molecular Angiogenesis, Interdisciplinary Cluster for Applied Genoproteomics (GIGA-Research), University of Liège, Liège, Belgium; and ${ }^{7}$ Department of Medicine, University of Washington, Seattle, Washington, United States of America

miR-155 has been shown to participate in host response to infection and neuroinflammation via negative regulation of blood-brain barrier (BBB) integrity and T cell function. We hypothesized that miR-155 may contribute to the pathogenesis of cerebral malaria (CM). To test this hypothesis, we used a genetic approach to modulate miR-155 expression in an experimental model of cerebral malaria (ECM). In addition, an engineered endothelialized microvessel system and serum samples from Ugandan children with CM were used to examine anti-miR-155 as a potential adjunctive therapeutic for severe malaria. Despite higher

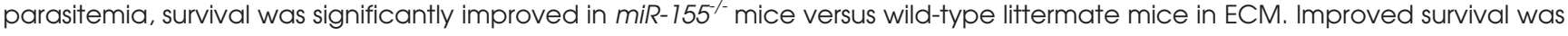
associated with preservation of BBB integrity and reduced endothelial activation, despite increased levels of proinflammatory cytokines. Pretreatment with antagomir-155 reduced vascular leak induced by human CM sera in an ex vivo endothelial microvessel model. These data provide evidence supporting a mechanistic role for miR-155 in host response to malaria via regulation of endothelial activation, microvascular leak and BBB dysfunction in CM.

Online address: http://www.molmed.org

doi: 10.2119/molmed.2016.00139

\section{INTRODUCTION}

There were an estimated 287 million cases of malaria and 731,500 deaths from the disease worldwide in 2015 (1,2). Plasmodium falciparum infection may progress to severe malaria (SM), a complex multisystem disorder associated with multiorgan dysfunction and a high mortality rate despite treatment with parenteral antimalarial drugs $(3,4)$. SM in children generally presents as one or more of the following syndromes: cerebral malaria (CM), severe malarial anemia (SMA) or acidosis/respiratory distress (RD).

The severity and outcome of malaria infection depends on a dynamic interplay

${ }^{\dagger} W C L$ and KCK contributed equally to this work.

Address correspondence to Kevin C Kain, SAR Laboratories, Sandra Rotman Centre for Global Health, MaRS Centre, 101 College Street, TMDT 10-360A, ON, M5G 1L7, Canada. Phone: + 1 (416) 581-7704. E-mail: kevin.kain@uhn.ca

Submitted May 26, 2016; Accepted for Publication January 26, 2017;

Published Online (www.molmed.org) February 2, 2017.

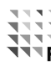

Feinstein Institute

for Medical Research

Northwell Health

between parasite virulence factors and host response determinants. A critical component of this response includes endothelial cell activation and dysfunction $(5,6)$. The endothelium is the largest interconnected organ in the body, and endothelial dysfunction, including breakdown of the blood-brain barrier (BBB), is a common pathway of injury in many life-threatening infections, including CM $(7,8)$. Markers of endothelial activation are associated with disease severity and predict outcome in both human and animal models of malaria (9-11).

The angiopoietin/receptor tyrosine kinase Tie2 (Ang/Tie2) system is a critical regulator of endothelial function and microvascular leak. Angiopoietin-1 (Ang-1) signals through its cognate 
receptor Tie2 to decrease inflammation and maintain endothelial quiescence through inhibition of nuclear factor kappa-light chain enhancer of activated B cells and reduced expression of cell adhesion molecules, including E-selectin expression $(12,13)$. This signaling also promotes cell survival through the phosphatidylinositol 3'kinase/AKT pathway, and vascular stabilization by inhibiting vascular endothelial growth factor (VEGF)-mediated leak $(14,15)$. Angiopoietin-2 (Ang-2) competes with Ang-1 for binding to Tie2, thereby promoting proinflammatory responses and microvascular leak $(16,17)$. Endothelial dysfunction appears to directly contribute to critical illness, multiorgan failure and death, and therefore represents an attractive target for interventions to improve outcome in CM and other life-threatening infections. However, the molecular mechanisms underlying endothelial activation and BBB dysfunction during $\mathrm{CM}$ are incompletely understood.

MicroRNAs (miRNAs) are a class of small noncoding RNA molecules approximately 18-25 nucleotides in length. miRNAs function as important posttranscriptional regulators of gene expression by pairing with the $3^{\prime}$ untranslated regions of target genes, thereby inhibiting translation and/or promoting degradation of mRNA (18). miRNAs, including miR-155, have been shown to regulate host response to apicomplexan parasites such as Toxoplasma (19). Brain endothelial miR-155 is also reported to negatively regulate $\mathrm{BBB}$ function during neuroinflammation associated with experimental autoimmune encephalomyelitis (EAE), a model of multiple sclerosis (MS), and systemic inflammation induced by lipopolysaccharide (20). Based on the above observations, we tested the hypothesis that miR-155 plays a mechanistic role in the pathogenesis of $\mathrm{CM}$.

\section{MATERIALS AND METHODS}

\section{Murine Model of ECM}

Plasmodium berghei ANKA ( $\mathrm{PbA}$ ) was obtained from the Malaria Resource
Centre (MR4) and maintained by passage in naïve C57BL / 6 donor mice. The University Health Network Animal Use Committee approved all experiments. bic/miR-155-deficient C57BL/ 6 albino mice were generated through $\mathrm{bic} / \mathrm{miR}-155$ null alleles as described (21). Mice were bred in-house and $m i R-155^{--}$and wildtype $(\mathrm{w} / \mathrm{t})$ littermate controls were used for experiments. C57BL/ $6 \mathrm{w} / \mathrm{t}$ mice were purchased from Jackson Laboratory and BALB/c mice were purchased from Charles River Laboratories. Mice were 6-10 wks old, mixed sex, weighing $17-28 \mathrm{~g}$ at the time of infection. Infection was initiated by intraperitoneal injection of $1 \times 10^{6} \mathrm{PbA}$-parasitized erythrocytes (PEs)/mouse and monitored daily for parasitemia on thin blood smears stained with Diff Quick (American Scientific Products). Mice were evaluated for signs of ECM (including limb paralysis, seizures and coma) and weight loss twice daily, and were euthanized when moribund.

\section{Assessment of BBB Integrity}

Evans Blue assay was used to assess BBB integrity as described (9). Brains were collected, photographed and placed in formamide for $48 \mathrm{~h}$ to extract Evans Blue. Evans Blue was quantified using a spectrophotometer at $605 \mathrm{~nm}$ and compared with a standard curve (Gen5, BioTek).

\section{Assessment of Systemic Cytokines and Markers of Endothelial Activation}

Heparinized plasma was collected via the saphenous vein at baseline ( $\mathrm{d} 0)$ and at $\mathrm{d} 6$ post $\mathrm{PbA}$ infection. Plasma cytokine levels were assessed using murine Cytometric Bead Array (BD Biosciences). Plasma levels of Ang-1, Ang-2, soluble Tie 2 (sTie2) and soluble E-selectin (sE-selectin) were determined by enzyme-linked immunosorbent assay (ELISA) (R\&D Systems) as described $(9,22)$.

\section{Assessment of Exosomal miR-155 Levels by Quantitative Reverse Transcription Polymerase Chain Reaction}

To isolate circulating exosomes, mouse blood was collected via cardiac puncture and exosomes were isolated from $100 \mu \mathrm{l}$ of plasma using ExoQuick Precipitation Solution (Systembio) as described (23).

\section{Assessment of Brain miR-155, mRNA Markers of Endothelial Activation, T cell Response and Oxidative Stress}

RNA was extracted from snap-frozen brain tissue after homogenization in TRIzol (1 mL/100 mg tissue) according to the manufacturer's protocol. RNA was extracted and cDNA was amplified and normalized by geometric averaging of glyceraldehyde 3-phosphate dehydrogenase, hypoxanthine-guanine phosphoribosyltransferase and $18 \mathrm{~S}$ expression levels as described (24). miR-155 relative expression was normalized to $U 6$ as described (25). The primer sequences used (5'-3') are shown in Supplementary Table S1.

\section{Patient Samples}

Ethical approval was granted by the Uganda National Council for Science and Technology, the Makerere University Research Ethics Committee and the Toronto Academic Health Science Network. The accompanying parent or caregiver of each study participant provided written informed consent. The study was conducted according to Declaration of Helsinki and International Council for Harmonisation guidelines on Good Clinical Practice. Serum samples were collected from children presenting to the Jinja Regional Pediatric Referral Hospital who met World Health Organization criteria for CM (26). Sera were collected at admission and stored at $-80^{\circ} \mathrm{C}$ until use.

\section{Assessment of Human CM Samples Using Microvessel Chambers}

Ex vivo microvessels were engineered in type I collagen as described (27). Human umbilical vein endothelial cells (HUVECs) were seeded through the microchannels, attached and cultured. Vessels were perfused with $80 \mu \mathrm{L}$ of either normal human sera, sera from CM patients or CM sera after preincubation with a $20 \mathrm{nM}$ mmu-miR-155 LNA inhibitor (miR-155 antagomir; Exiqon) or negative control "scramble" (Exiqon). As a 
measure of permeability, $40 \mathrm{kDa}$ fluorescein isothyocyanate-conjugated Dextran was used, and the corresponding permeability coefficient of the endothelium, $K(\mu \mathrm{m} / \mathrm{s})$, was measured. Immunostaining with CD31 (Abcam) and Hoechst 33342 (Abcam) was done to examine the junctions at cell contacts (see Supplementary Data for detailed methods).

\section{Statistical Analysis}

Statistical analysis was performed using GraphPad Prism software version 6.07 (GraphPad Software). Statistical significance for survival studies was assessed by log-rank test. Parasitemia was assessed by a random-intercept, random-slope, linear mixed-effects model using $\mathrm{R}$ version 3.0.1 (R Foundation for Statistical Computing). After tests for normality, other comparisons were assessed by either two-tailed unpaired $t$ test with Welch's correction/Mann-Whitney test, or one-way analysis of variance (ANOVA)/Kruskal-Wallis test, or twoway ANOVA $(\alpha=0.05)$ with post hoc tests where appropriate. Differences were considered significant when $P<0.05$.

All supplementary materials are available online at www.molmed.org.

\section{RESULTS}

\section{Circulating Exosomal miR-155 Levels Increased over the Course of ECM and Were Associated with ECM Susceptibility}

Inbred mouse lines with varying susceptibility to malaria can be powerful tools to map genetic determinants regulating host response to human infection $(28,29)$. To begin to investigate the potential relationship between miR-155 and susceptibility to ECM, we examined the kinetics of circulating exosomal miR-155 in C57BL/ 6 mice displaying marked susceptibility to ECM compared with BALB/c mice, which are more resistant to $\mathrm{PbA}$-induced ECM (30). The kinetics of $\mathrm{PbA}$ infection in these strains show that C57BL/ 6 mice are more susceptible to $\mathrm{PbA}$ infection than $\mathrm{BALB} / \mathrm{c}$ mice
$(P<0.0001 ;$ Figure 1A). Relative circulating levels of exosomal miR-155 in peripheral blood were determined by quantitative reverse transcription polymerase chain reaction (qRT-PCR) (Figure 1B). Analysis with two-way ANOVA with post hoc test revealed a significant interaction between the effects of strain and time $(P<0.0001)$. Exosomal miR-155 levels were higher in C57BL/ 6 than BALB/c mice at $\mathrm{d} 5$ of $\mathrm{PbA}$ infection $(P<0.0001)$ immediately before the onset of ECM (d 6-10). Over the course of infection in C57BL/ 6 mice, there were significantly higher levels at d 5 versus $d 1$ $(P<0.0001)$ and d $0(P<0.0001)$, which subsequently decreased to near basal levels by d $6(P<0.0001)$. Relative brain miR-155 levels were also determined by qRT-PCR, but we found no differences between brain miR-155 levels over the course of $\mathrm{PbA}$ infection $(P>0.05$; two-way ANOVA).

miR-155 Deficiency Is Associated with Improved Survival and Preservation of BBB Integrity in ECM

To further investigate a potential mechanistic role for miR-155, we

A

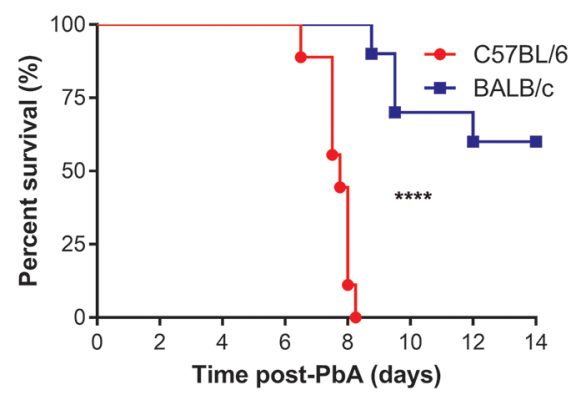
compared survival in the ECM model

following $\mathrm{PbA}$ infection in $\mathrm{miR}-155^{-/}$ albino C57BL/ 6 mice. Compared to $\mathrm{w} / \mathrm{t}$ littermates, survival was significantly improved in $m i R-155^{--}$mice $(P<0.0001$; Figure 2). We then investigated whether improved survival was associated with

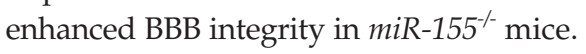
Based on Evans Blue assay as a marker of microvascular leak, BBB integrity was better preserved in $\mathrm{PbA}$-infected miR-155 ${ }^{-/}$ mice versus $\mathrm{PbA}$-infected $\mathrm{w} / \mathrm{t}$ littermate controls $(P<0.0001$; Figures 3A, B). Of note, there was enhanced survival and BBB integrity despite higher observed peripheral parasitemia in $\mathrm{miR}-155^{-/}$ mice versus $\mathrm{w} / \mathrm{t}$ mice (Figure $3 \mathrm{C}$ ). In a random-intercept, random-slope, linear mixed-effects model, the parasitemia increased by $5.4 \%$ (95\% confidence interval [Cl] 4.3-6.5) per day on average in the $\mathrm{w} / \mathrm{t}$ group and by $10.4 \%(95 \% \mathrm{Cl}$ 7.9-12.9) per day in the $m i R-155^{-/-}$group $(P<0.0001$; Figure 2B).

\section{PbA-Infected miR-155/- Mice Have Increased Inflammation But Decreased Endothelial Activation in Response to Infection}

Inflammatory responses and endothelial activation have been implicated in

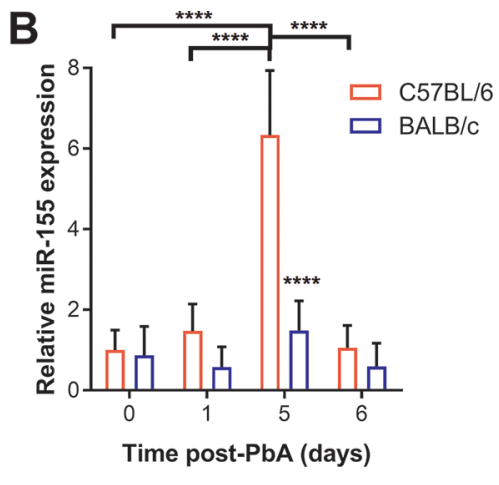

Figure 1. Circulating exosomal miR-155 levels increased over the course of experimental cerebral malaria (ECM) and were associated with ECM susceptibility in $P$. berghei ANKA ( $\mathrm{PbA}$ ) infection. (A) C57BL/6 mice were more susceptible to $\mathrm{PbA}$ infection than BALB/c mice. Survival of C57BL/6 mice was compared with BALB/c mice infected with $1 \times 10^{5}$ parasitized erythrocytes of $\mathrm{PbA}$. Survival was significantly less in PbA-infected C57BL/6 than BALB/c mice ${ }^{* * *} P<0.0001$; log-rank test $n=9-10 /$ group). (B) Exosomal miR-155 levels increased over the course of infection in ECM-susceptible mice. Median circulating exosomal miR-155 levels, measured by qRT-PCR, were significantly higher in C57BL/6 than BALB/C mice at d 5, immediately before the onset of ECM $\left({ }^{* * * *} P<0.0001\right.$; two-way ANOVA with Tukey's multiple comparisons test; $n=4-5$ /group). Error bars represent standard deviation. 
the pathophysiology of both human and murine CM (5,31-33). We examined the impact of miR-155 deficiency on markers of inflammation and endothelial activation

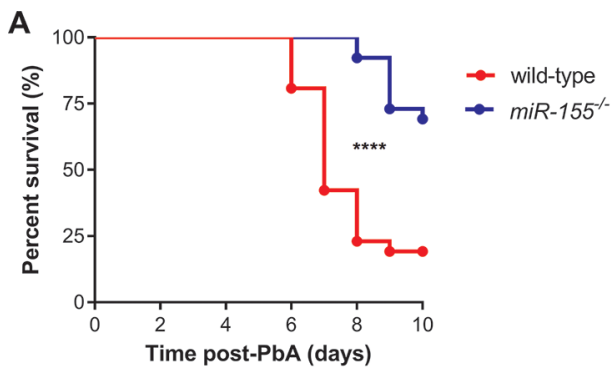

following PbA infection. Plasma endothelial activation markers were measured in uninfected and PbA-infected miR-155 mice and littermate controls (Figure 4,

Figure 2. miR-155 deficiency was associated with improved survival despite increased peripheral parasitemia in $P$. berghei ANKA (PbA)-induced ECM. (A) Survival was improved in

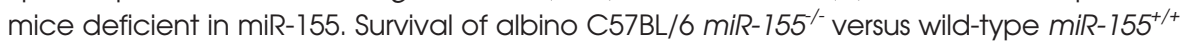
littermate controls infected with $1 \times 10^{6}$ parasitized erythrocytes was compared, and miR-

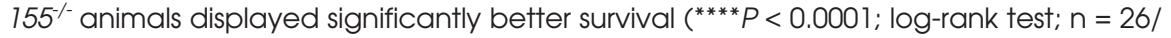
group). (B) Peripheral parasitemia was greater in mice deficient in miR-155. Mean peripheral parasitemia in miR- $155^{-/}$versus wild-type mice infected with $1 \times 10^{6}$ parasitized erythro-

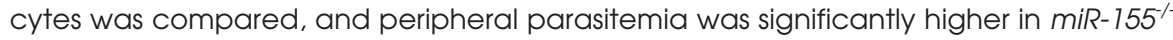
than wild-type mice ( ${ }^{* * *} P<0.0001$; linear mixed-effects model; $n=26 /$ group). Experiment was repeated for confirmation of findings. Error bars represent standard deviation.
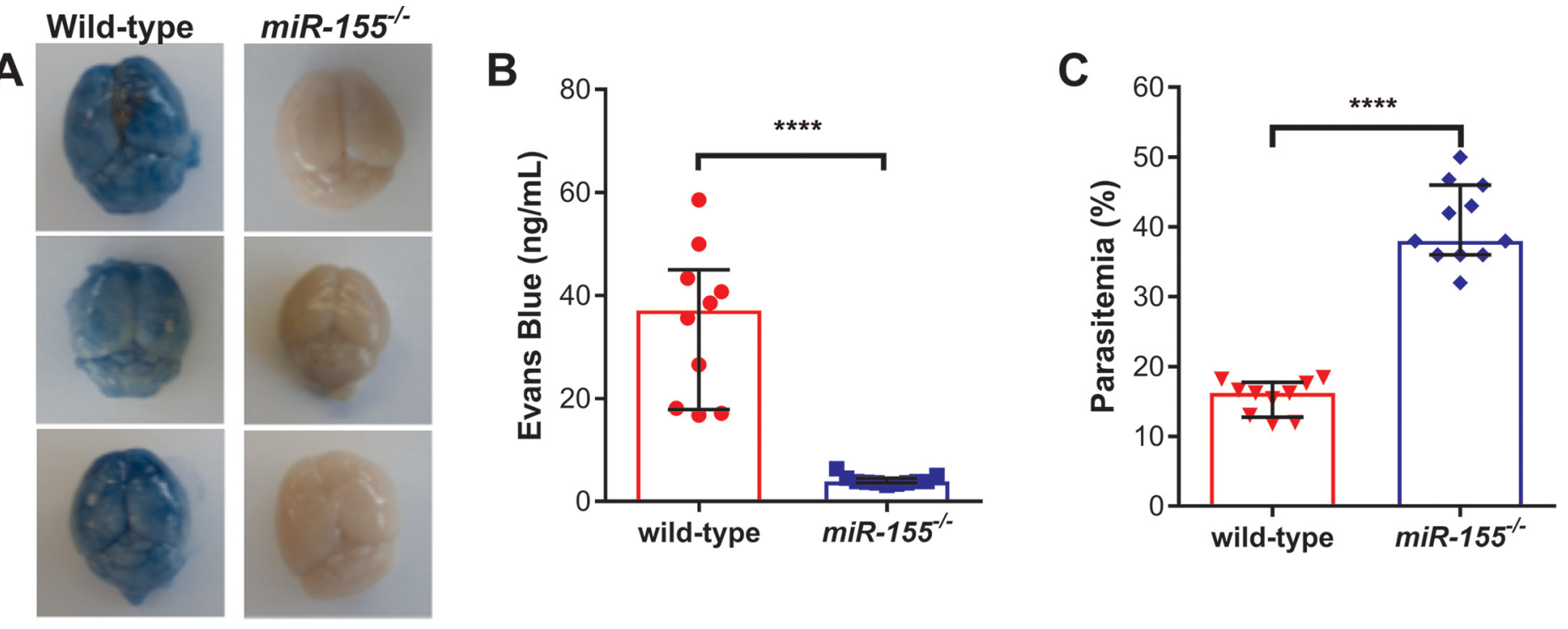

Figure 3. miR-155 deficiency was associated with preservation of the blood-brain barrier (BBB) in $P$. berghei ANKA (PbA)-induced ECM. (A) Brains from mice deficient in miR-155 showed improved BBB integrity (less dye leakage into the brain parenchyma). Evans Blue assay

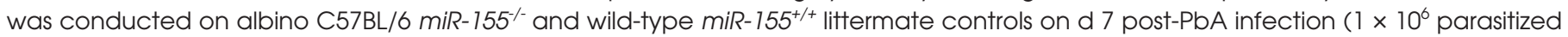
erythrocytes; $n=10-11$ /group), and representative photographs of brains from mice following Evans Blue dye assay are shown. (B) Brain microvascular leak was reduced in miR-155-deficient mice. Median levels of Evans Blue in the brain was quantified as an indication

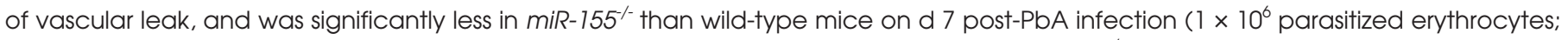
${ }^{* * * *} P<0.0001$; Mann-Whitney test; $\mathrm{n}=10-11$ /group). (C) BBB integrity was preserved in miR-155\% mice despite increased peripheral parasitemia. Median peripheral parasitemia at the time of Evans Blue assay was measured in miR- $155^{-1}$ and wild-type mice $\left({ }^{* * * *} P<0.0001\right.$; Mann-Whitney test; $n=10-11$ /group). Experiment was repeated for confirmation of findings. Error bars represent interquartile range. 

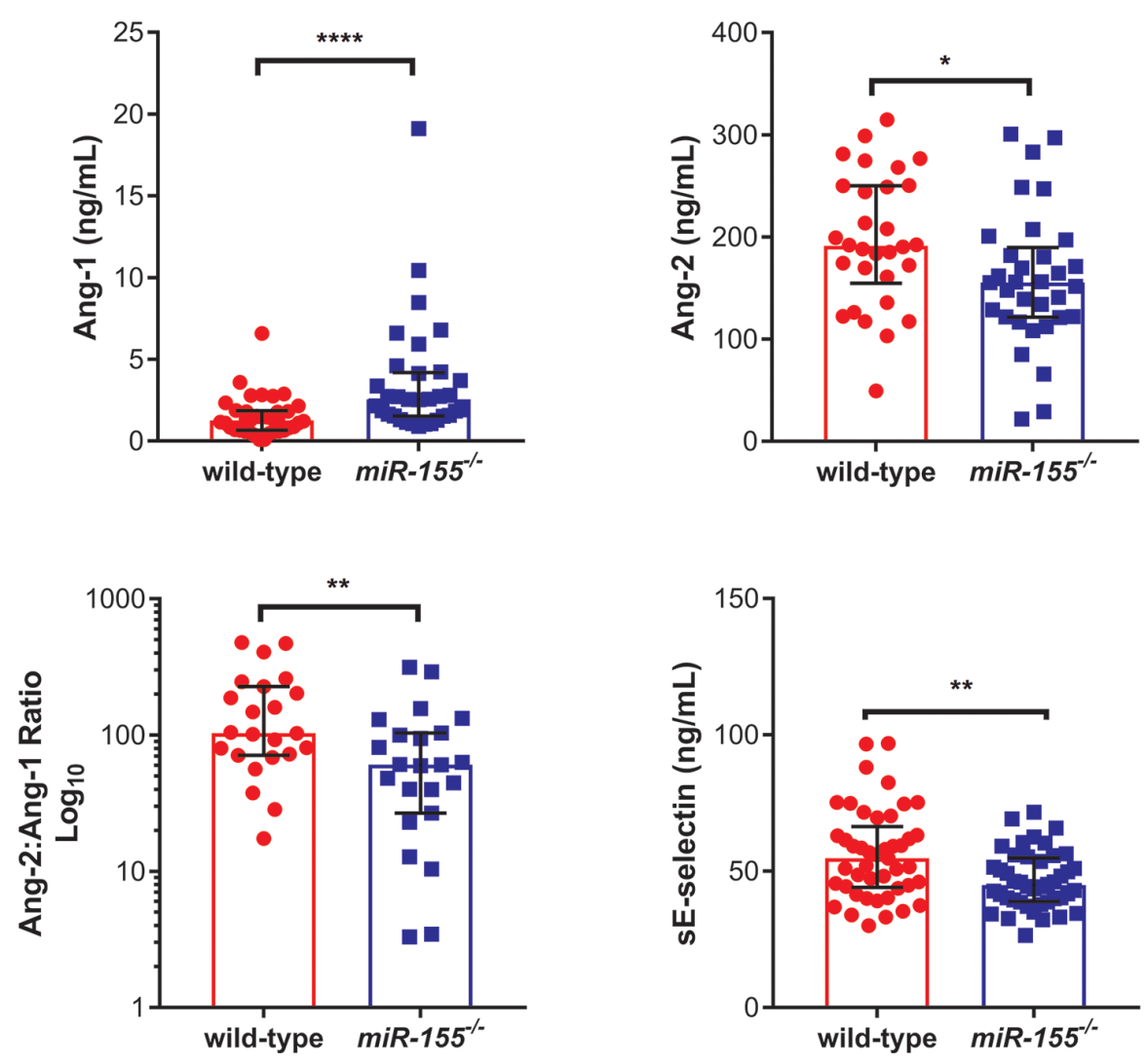

Figure 4. miR-155 deficiency is associated with decreased endothelial activation during $P$. berghei ANKA (PbA)-induced ECM. Median plasma protein levels of endothelial ac-

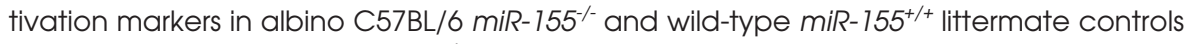
on d 6 post-PbA infection ( $1 \times 10^{6}$ parasitized erythrocytes), measured by ELISA. Ang- 1 was significantly higher, while Ang-2, Ang-2:Ang- 1 ratio and sE-selectin were significantly

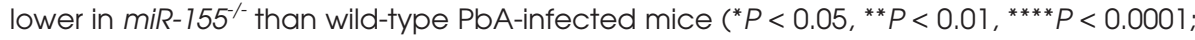
Welch's $t$ test or Mann-Whitney test, where applicable; $n=23-45 /$ group). Experiment was repeated for confirmation of findings. Error bars represent interquartile range. Abbreviations: Ang-1, angiopoietin-1; Ang-2, angiopoietin-2; sE-selectin, soluble E-selectin.

of Gzma, a marker of cytotoxic T lymphocytes and natural killer cells, was significantly decreased in the brains of miR $-155^{-/}$infected mice versus $\mathrm{w} / \mathrm{t}$ controls $(P<0.05$; Figure 6$)$. Similarly, brain expression of $\mathrm{Ho}-1$, a marker of oxidative stress, was significantly decreased in $\mathrm{PbA}$-infected $\mathrm{miR}-155^{-/}$mice versus $\mathrm{w} / \mathrm{t}$ mice $(P<0.05$; Figure 6$)$.

\section{miR-155 Inhibition with an Antagomir Reduces Vascular Leak in Microvessels Exposed to Sera from Ugandan Children with CM}

To extend our observations to human $\mathrm{CM}$ and to assess a functional strategy to disrupt miR-155 function using antagomirs (oligonucleotides that are specific and efficient silencers of miRNA [34]), we examined microvascular leak in a human endothelial microvessel model using sera from Ugandan children with CM. Sera from two children with CM or normal control sera were perfused through microvessel networks that had been preincubated with miR-155 antagomir or a negative "scrambled" control (Figure 7). Using fluorescein isothyocyanate-dextran to assess microvascular leak, CM sera induced greater leak $(K=0.26 \pm 0.13 \mu \mathrm{m} / \mathrm{s})$ than normal control sera $(K=0.016 \pm 0.005 \mu \mathrm{m} / \mathrm{s})$. Compared to scrambled control, preincubation with miR-155 antagomir resulted in decreased microvascular leak to near basal levels $(K=0.065 \pm 0.029 \mu \mathrm{m} / \mathrm{s})$, supporting a role for miR-155 in microvascular leak (Figures 7B,C, Supplementary Videos 1-3).

\section{DISCUSSION}

Endothelial and BBB dysfunction is a central feature of $\mathrm{CM}$ in humans and ECM in murine models $(9,35,36)$. In this study, we used a combination of in vivo preclinical models of ECM to provide direct evidence for a role for miR-155 as a negative regulator of endothelial and BBB integrity during severe malaria. We supplemented this in vivo evidence using an ex vivo human microvessel model. In the ECM murine model, genetic deletion of miR-155 resulted in a significantly improved survival, decreased endothelial activation and preservation of the BBB despite higher parasite burden and increased systemic inflammation. In the ex vivo microvessel model, an antagomir to miR-155 reduced microvascular leak induced by sera from children with CM.

There are several potential pathways by which miR-155 may modulate microvascular integrity, including effects on VE-cadherin and annexin-A2 (ANXA2). miR-155 has been shown to target ANXA2, which binds to VE-cadherin and is required for endothelial barrier function $(20,37)$. Genetic or functional disruption of miR-155 may improve endothelial and BBB integrity, at least in part, by preventing miR-155-induced downregulation of ANXA2. Our observations are consistent with reports of other neuroinflammatory syndromes associated with increased BBB permeability, such as the EAE animal model of MS (20). In these studies, miR-155 was highly expressed in active MS lesions associated with BBB breakdown. Genetic deletion of $m i R-155$ decreased BBB leak in murine EAE models, and endogenous inhibition of miR-155 decreased cytokine-induced endothelial permeability via modulation of endothelial cell-cell and cell-matrix interactions (20). 

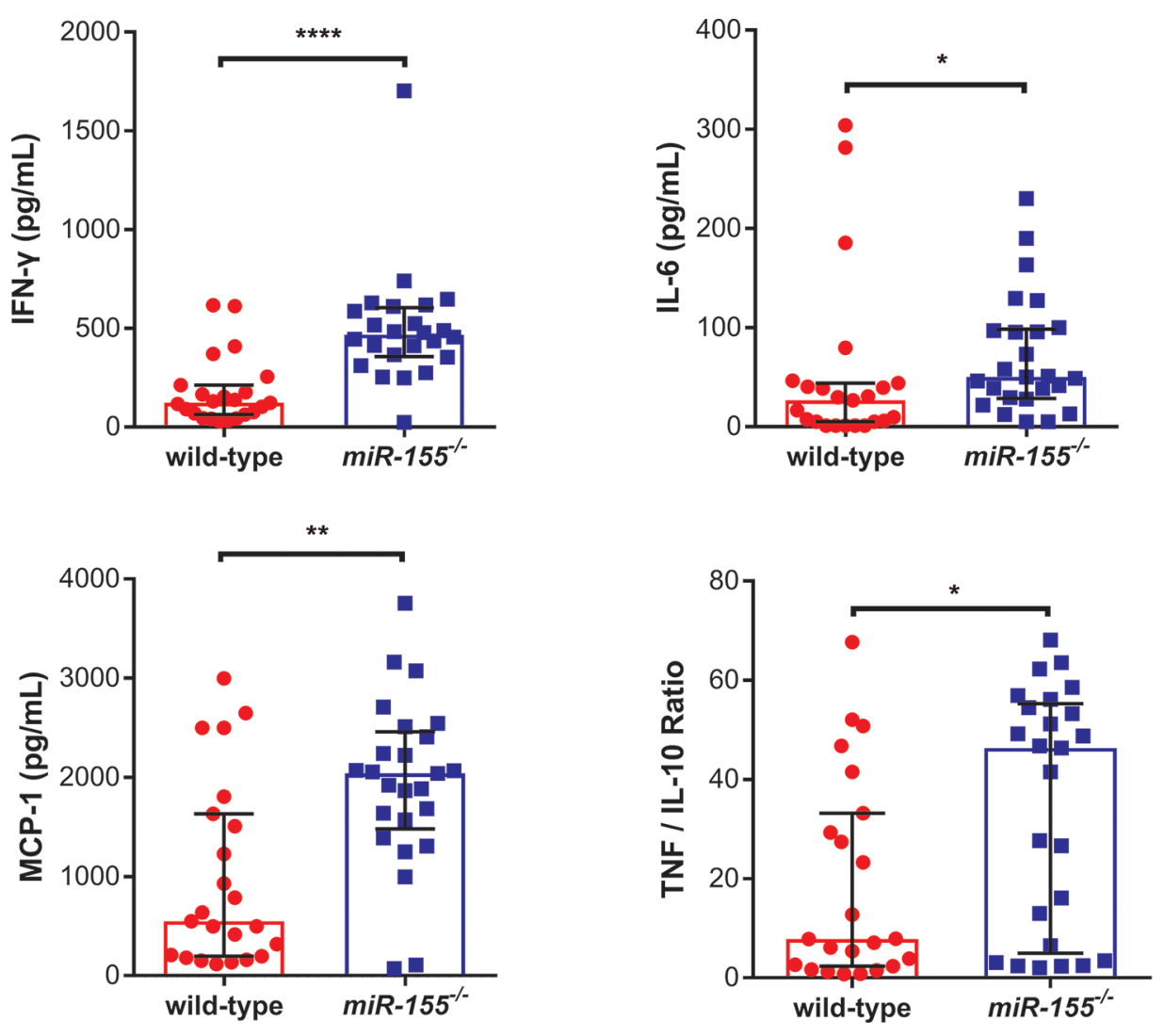

Figure 5. miR-155 deficiency is associated with increased inflammation during $P$. berghei ANKA (PbA)-induced ECM. Median plasma protein levels of inflammatory cytokine mark-

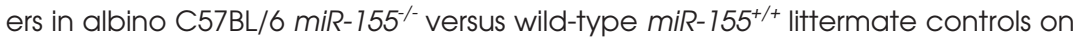
d 6 post- $\mathrm{PbA}$ infection $\left(1 \times 10^{6}\right.$ parasitized erythrocytes), measured by Cytometric Bead

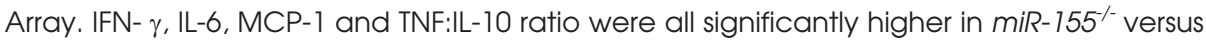
wild-type PbA-infected mice $\left({ }^{*} P<0.05,{ }^{* *} P<0.01,{ }^{* * *} P<0.0001\right.$; Mann-Whitney test; $n=23-25$ /group). Experiment was repeated for confirmation of findings. Error bars represent interquartile range. Abbreviations: IFN- $\gamma$, interferon-gamma; TNF, tumor necrosis factor; IL-6, interleukin-6; MCP-1, monocyte chemotactic-1; IL-10, interleukin-10.

Our study also examined the effect of miR-155 on other pathways implicated in endothelial activation and microvascular integrity. Levels of exosomal miR-155 are reported to be a good determinant of its functionality (38). Therefore we assessed whether ECM susceptibility in PbAinfected $\mathrm{w} / \mathrm{t}$ mice corresponded to circulating exosomal miR-155 levels. We observed increases in circulating exosomal levels of miR-155 only in ECM-susceptible mice (for example, C57BL/6) and just prior to the onset of ECM. Exosomes containing miRNA can be disseminated via the circulation, delivering miRNA and regulating protein levels of its target genes at distal sites $(39,40)$. Susceptibility to ECM in C57BL/ 6 mice has been associated with enhanced endothelial activation, so we speculate that this may, in part, be associated with miR-155 levels $(9,32,41)$. In the $m i R-155^{-/}$model used in this study, compared with their wildtype counterparts, the endothelium was less activated during $\mathrm{PbA}$ infection, as evidenced by increased circulating Ang-1 and decreased Ang-2, Ang-2:Ang-1 ratio

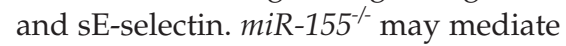
these effects by increasing Ang- 1 expression and decreasing expression of Ang-2 and adhesion molecules, although we did not find mRNA evidence to support this (Supplementary Figure S3). Nonetheless, our observations are consistent with the established role of the Ang-Tie2 axis as a critical regulator of endothelial activation, and the association of this pathway with disease severity and poor outcome in human malaria as well as the murine model of ECM $(16,17,32,42)$.

Increased Ho- 1 expression has previously been associated with protection in ECM (43). However, there is some controversy about its role, and Ho- 1 levels have been shown to correlate with disease severity in Gambian children with malaria (44) and brain levels with disease severity (45). In the present study, miR- $155^{--}$infected mice also had significantly lower Ho-1 mRNA levels than $\mathrm{w} / \mathrm{t}$ mice at $\mathrm{d} 6$ post-infection. This finding is consistent with a previous study of ECM, where Ho-1 mRNA expression was found to be progressively and significantly elevated through the course of infection in multiple regions of the brain in ECM-susceptible mice (46). Even if HO-1 is associated with protection in the ECM model, this association may be lost in the absence of miR-155. Recent studies using exosomal delivery of miR-155 reported increased expression of Ho- 1 mediated by miR-155 repression of Bach-1, an established transcriptional regulator of $\mathrm{Ho}-1$ (40,47-49). Collectively, these data are consistent with the hypothesis that in the absence of miR-155, there is increased Bach-1 expression, leading to decreased $\mathrm{Ho}_{\mathrm{O}} \mathrm{-}$ expression. Nonetheless, in miR-155 knockout animals with low Ho-1 levels, the integrity of the $\mathrm{BBB}$ is maintained, thus excluding an essential role for HO-1 in BBB integrity and survival in this model.

An additional pathway targeted by miR-155 is inflammation. In this study, genetic disruption of miR-155 was associated with increased circulating levels of proinflammatory cytokines and increased parasitemia during $\mathrm{PbA}$ infection. Conflicting roles for miR-155 in inflammation have been described (50). miR-155 has been shown to target suppressor of cytokine signaling 1 , thereby modulating IFN- $\gamma$ signaling, but this miRNA has also been proposed to 

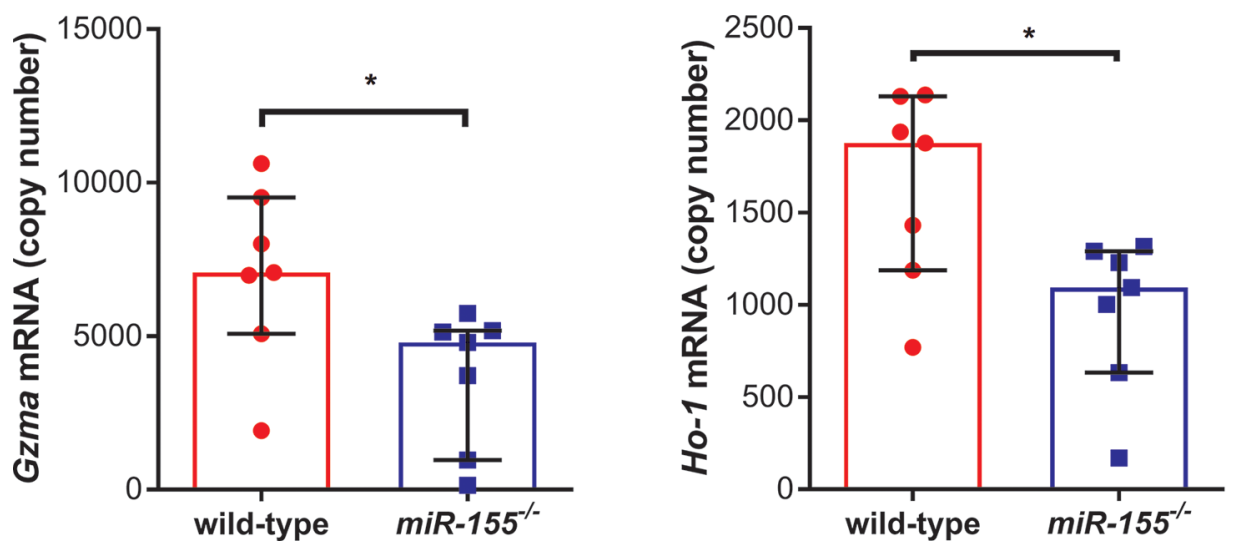

Figure 6. miR-155 deficiency is associated with decreased expression of granzyme A (a marker of cytotoxic T lymphocytes and natural killer cells) and heme oxygenase- 1 (a marker of oxidative stress) in response to $P$. berghei ANKA (PbA)-induced ECM. Median brain mRNA levels in albino C57BL/6 miR- $155^{-1}$ versus wild-type miR- $155^{+/+}$littermate controls on a 6 post- $\mathrm{PbA}$ infection $\left(1 \times 10^{6}\right.$ parasitized erythrocytes), measured by qRT-PCR. Gzma and Ho- 7 mRNA were significantly decreased in miR-155-- versus wildtype $\mathrm{PbA}$-infected mice ( ${ }^{*} P<0.05$; Mann-Whitney test; $\mathrm{n}=7$ /group). Experiment was repeated for confirmation of findings. Error bars represent interquartile range. Abbreviations: Gzma, granzyme A; Ho-1, heme oxygenase-1.

suppress the IFN- $\gamma$ receptor (51). Nonetheless, the beneficial effect of enhanced BBB integrity on survival appears to effectively counter the potential negative impact of increased parasitemia and systemic inflammation observed in these mice.

miR-155 has also been reported to regulate $\mathrm{T}$ cell trafficking and function (51-53). T cells have been shown to accumulate in the brain and to contribute to endothelial injury and death associated with ECM (54). PbA-infected miR-155/mice had decreased GZMA mRNA (a tryptase contained in cytotoxic $\mathrm{CD} 8^{+} \mathrm{T}$ cells that induces cell death) levels in the brain. These findings are consistent with studies examining the association of sphingosine 1-phosphate with ECM and human CM through lymphocyte trafficking (22). Sphingosine 1-phosphate modulation using FTY720 has been shown to decrease brain $\mathrm{CD} 4^{+} / \mathrm{CD} 8^{+} \mathrm{T}$ cells and Gzma mRNA, contributing to decreased endothelial activation, enhanced BBB integrity and improved survival (22). Using GZMA as a marker for $\mathrm{CD}^{+} \mathrm{T}$ cell accumulation and activation in the brain, our data are consistent with recent evidence that altered $\mathrm{T}$ cell subsets, including effector $\mathrm{CD}^{+} \mathrm{T}$ cells, may play a causal role in the pathogenesis of ECM, and that $\mathrm{T}$ cell responses can be altered by the modulation of miR-155 $(52,53,55)$. Depletion of $\mathrm{CD}^{-} \alpha^{+}$dendritic cells in $\mathrm{PbA}$-infected mice has been shown to delay lethality, reduce brain pathology and deplete $\mathrm{CD} 8^{+}$accumulation, without affecting peripheral parasitemia (56). We propose that the high peripheral parasitemia observed in $m i R-155^{-/-}$mice may be due, at least in part, to decreased $\mathrm{T}$ cell activation and differentiation, limiting immune mechanisms that control parasitemia $(21,57)$.

There are limitations to this study. Although the Ang-1 plasma levels are consistent with other murine and human studies of cerebral malaria, the Ang-2 plasma levels are higher than in human studies $(22,45,58)$, The Ang-2 ELISA is a new (but validated) assay, and there are less published data; however, the levels observed in our uninfected mice are in agreement with the manufacturer's expected ranges for plasma, and our group consistently obtained the same range of plasma Ang-2 levels. Although this limitation should be taken into consideration as impacting the Ang2:Ang-1 ratio, the relative changes in these factors during malaria infection of mice or humans remain similar, and disease severity and death are associated with relative decreases in Ang-1 levels and relative increases in Ang-2 between these analytes. The use of organ-specific microvascular endothelium, such as human brain microvascular endothelial cells in the microvessel model, may better reflect the effect of circulating mediators found in CM on brain endothelial cells. The microvessel experiments using human CM sera support the hypothesis that miR-155 may also play a role in the pathogenesis of human CM. The focus of this study was to examine the mechanistic role of miR-155 in host response to malaria infection; however, future studies are needed to explore potential therapeutic roles of miR-155 antagomirs in combination with antimalarials.

\section{CONCLUSION}

In summary, we have shown in a preclinical model of cerebral malaria that genetic deletion of miR-155 enhances endothelial quiescence and BBB integrity and survival. We suggest that these observations are associated with dysregulation of the Ang/Tie2 axis. Administration of a miR-155 antagomir was able to preserve endothelial integrity and reduce microvascular leak induced by serum samples from two human CM cases in an ex vivo endothelial microvessel system. miR-155 has multiple targets and transcriptional effects, and additional studies will be required to delineate its complex role in regulating host response and endothelial integrity. Further investigation of therapeutic strategies to inhibit the function of miR-155 and improve clinical outcomes in CM are warranted.

\section{ACKNOWLEDGMENTS}

This study was supported in part by the Canadian Institutes of Health Research (CIHR MOP-13721, MOP-136813 and MOP-115160 and a CIHR Foundation 

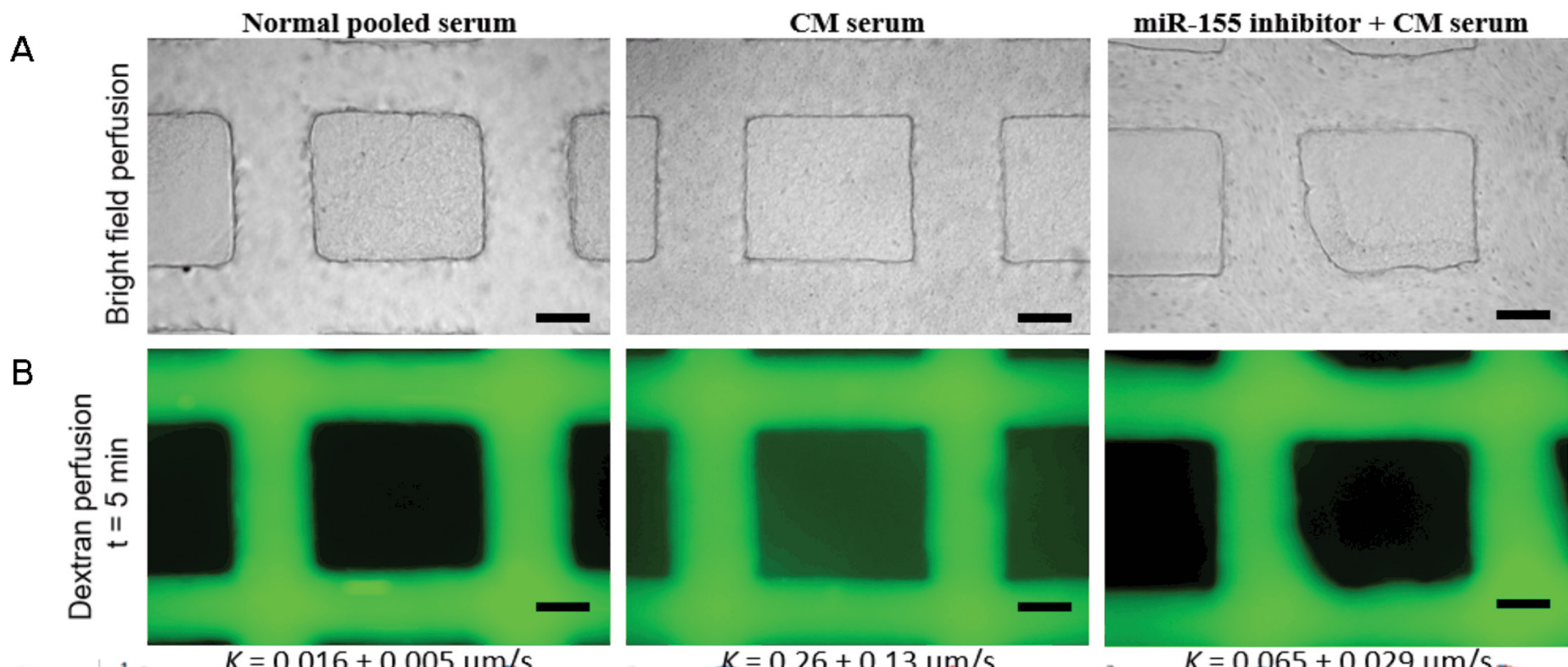

C

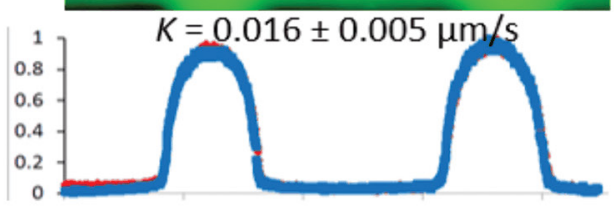

D
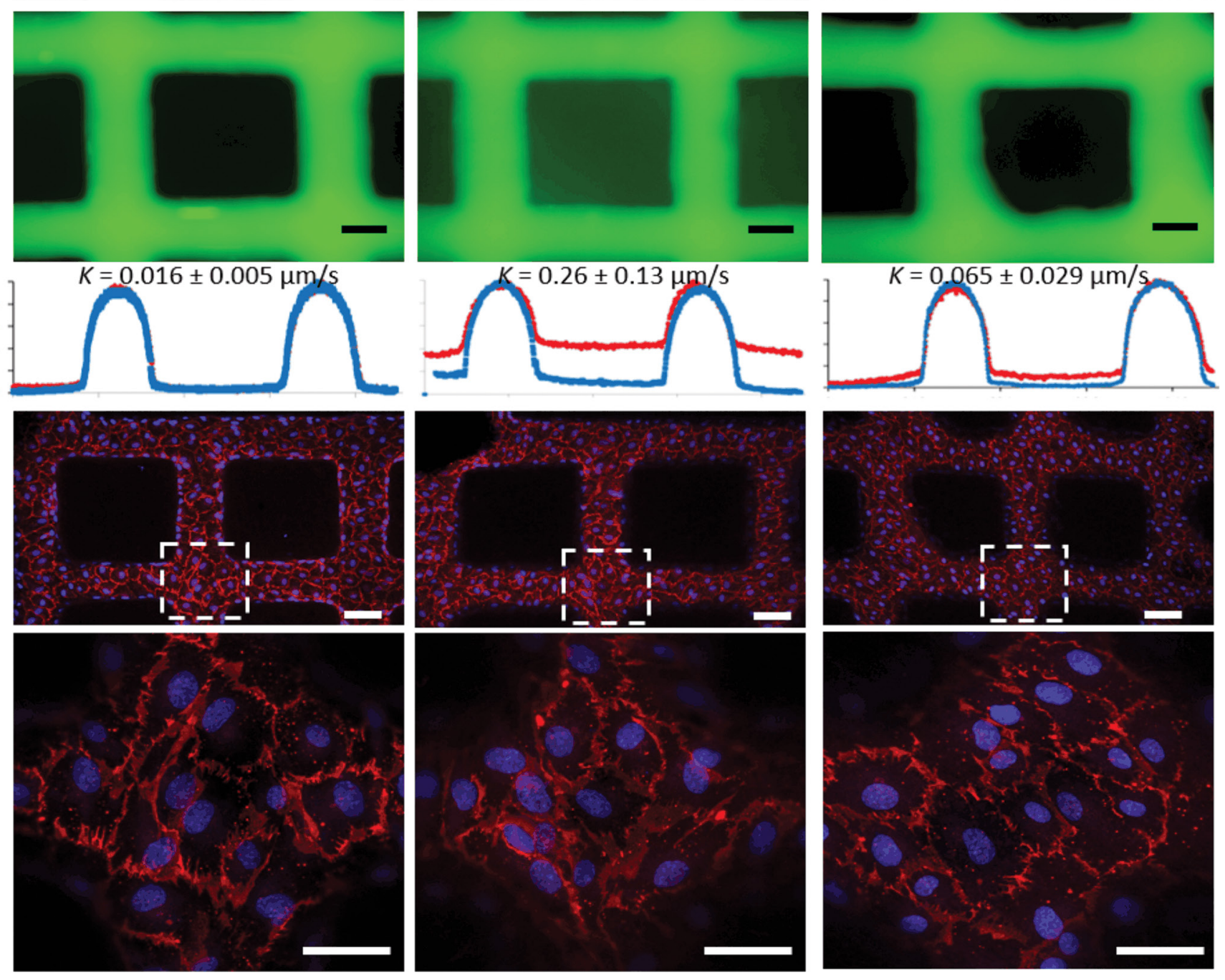

Figure 7. Human umbilical vein endothelial cell (HUVEC) microvessels perfused with sera from children with cerebral malaria (CM) illustrate greater dextran leak versus sera control, which can be prevented with preincubation with miR-155 antagomir. (A) Bright field image of human sera perfused through the microvessels: normal pooled sera (left panel), CM sera (middle panel) and microvessels pretreated with antagomir-155 prior to $\mathrm{CM}$ sera (right panel) (scale bar: $100 \mu \mathrm{m}$ ). (B). Fluorescence image of microvessels perfused with $40 \mathrm{kDa}$ dextran (as a marker of microvascular leak) after 5 min of perfusion, after different sera treatments. (C) Line cut of intensity profile of dextran-perfused microvessels in B at $t=1 \mathrm{~min}$ (blue line) and $t=5 \mathrm{~min}$ (red line). The permeability $\mathrm{K}$ of three conditions was determined to be $0.016 \pm 0.005 \mu \mathrm{m} / \mathrm{s}$ (normal sera), $0.26 \pm 0.13 \mu \mathrm{m} / \mathrm{s}(\mathrm{CM}$ sera) and $0.065 \pm 0.029 \mu \mathrm{m} / \mathrm{s}$ (antagomir-155 + CM sera).

(D) Z-stack projection of confocal fluorescence images of microvessels after serum treatment and dextran perfusion, stained with CD31 (cell junction) and Hoechst 33342 (nuclei) (scale bar: $100 \mu \mathrm{m}$ ). Bottom panels: zoomed-in view of dashed square box (scale bar: $50 \mu \mathrm{m}$ ). Confocal images illustrate no differences in PECAM1/CD31 cell junctions despite significant differences in dextran leak. The assay was repeated with a different CM sample and yielded similar results ( $n=2$ biological replicates). 
grant to KCK); Canadian Vascular Network Seed Funding and CIHR (MOP-119506 to JEF); Canadian Research Chairs (to KCK, WCL and JEF); and kind donations from the Tesari Foundation and Kim Kertland. Studentship provided by Peterborough KM Hunter Charitable Foundation Graduate Awards and the McCuaig-Throop Bursary (to KRB) and Canadian Vascular Network Scholarships (to HSC and MSN). We thank Dr. Lena Serghides for technical expertise.

\section{DISCLOSURE}

The authors declare they have no competing interests as defined by Molecular Medicine or other interests that might be perceived to influence the results and discussion reported in this paper.

\section{REFERENCES}

1. GBD 2015 Disease and Injury Incidence and Prevalence Collaborators. (2016) Global, regional, and national incidence, prevalence, and years lived with disability for 310 diseases and injuries, 19902015: a systematic analysis for the Global Burden of Disease Study 2015. Lancet. 388:1545-1602.

2. Wang H, et al. (2016) Global, regional, and national life expectancy, all-cause mortality, and cause-specific mortality for 249 causes of death, 1980-2015: a systematic analysis for the Global Burden of Disease Study 2015. Lancet. 388:1459-1544.

3. Dondorp AM, et al. (2010) Artesunate versus quinine in the treatment of severe falciparum malaria in African children (AQUAMAT): an open-label, randomised trial. Lancet. 376:1647-57.

4. Dondorp A, Nosten F, Stepniewska K, Day N, White N. (2010) Artesunate versus quinine for treatment of severe falciparum malaria: a randomised trial. Lancet. 366:717-25.

5. Kim H, Higgins S, Liles WC, Kain KC. (2011) Endothelial activation and dysregulation in malaria: a potential target for novel therapeutics. Curr. Opin. Hematol. 18:177-85.

6. Miller LH, Ackerman HC, Su X, Wellems TE. (2013) Malaria biology and disease pathogenesis: insights for new treatments. Nat. Med. 19:156-67.

7. Lee WL, Liles WC (2011) Endothelial activation, dysfunction and permeability during severe infections. Curr. Opin. Hematol. 18:191-6.

8. Page A V, Liles WC (2013) Biomarkers of endothelial activation/dysfunction in infectious diseases. Virulence. 4:507-16.

9. Serghides L, et al. (2011) Inhaled nitric oxide reduces endothelial activation and parasite accumulation in the brain, and enhances survival in experimental cerebral malaria. PLoS One. 6:e27714.
10. Yeo TW, et al. (2008) Angiopoietin-2 is associated with decreased endothelial nitric oxide and poor clinical outcome in severe falciparum malaria. Proc. Natl. Acad. Sci. USA. 105:17097-102.

11. Erdman LK, et al. (2011) Combinations of host biomarkers predict mortality among Ugandan children with severe malaria: a retrospective case-control study. PLoS One. 6:e17440.

12. Hughes DP, Marron MB, Brindle NPJ. (2003) The antiinflammatory endothelial tyrosine kinase Tie2 interacts with a novel nuclear factor-kappaB inhibitor ABIN-2. Circ. Res. 92:630-36.

13. Kim I, et al. (2001) Vascular endothelial growth factor expression of intercellular adhesion molecule 1 (ICAM-1), vascular cell adhesion molecule 1 (VCAM-1), and E-selectin through nuclear factor-kappa B activation in endothelial cells. J. Biol. Chem. 276:7614-20.

14. Gavard J, Patel V, Gutkind JS. (2008) Angiopoietin-1 prevents VEGF-induced endothelial permeability by sequestering Src through mDia. Dev. Cell. 14:25-36.

15. Kontos CD, Cha EH, York JD, Peters KG. (2002) The endothelial receptor tyrosine kinase Tie1 activates phosphatidylinositol 3-kinase and Akt to inhibit apoptosis. Mol. Cell. Biol. 22: 1704-13.

16. Fiedler U, et al. (2006) Angiopoietin-2 sensitizes endothelial cells to TNF-alpha and has a crucial role in the induction of inflammation. Nat. Med. 12:235-9.

17. Parikh SM, et al. (2006) Excess circulating angiopoietin-2 may contribute to pulmonary vascular leak in sepsis in humans. PLoS Med. 3:e46.

18. Engels BM, Hutvagner G. (2006) Principles and effects of microRNA-mediated post-transcriptional gene regulation. Oncogene. 25:6163-9.

19. Cannella D, et al. (2014) miR-146a and miR-155 delineate a MicroRNA fingerprint associated with Toxoplasma persistence in the host brain. Cell Rep. 6:928-37.

20. Lopez-Ramirez MA, et al. (2014) MicroRNA-155 negatively affects blood-brain barrier function during neuroinflammation. FASEB J. 28:2551-65.

21. Rodriguez A, et al. (2007) Requirement of bic/ microRNA-155 for normal immune function. Science. 316:608-11.

22. Finney CA, et al. (2011) S1P is associated with protection in human and experimental cerebral malaria. Mol. Med. 17:717-25.

23. Njock M-S, et al. (2015) Endothelial cells suppress monocyte activation through secretion of extracellular vesicles containing anti-inflammatory microRNAs. Blood. 125:3202-12.

24. Vandesompele J, et al. (2002) Accurate normalization of real-time quantitative RT-PCR data by geometric averaging of multiple internal control genes. Genome Biol. 3:RESEARCH0034.

25. Cheng HS, et al. (2013) MicroRNA-146 represses endothelial activation by inhibiting pro-inflammatory pathways. EMBO Mol. Med. 5:949-66.
26. Hawkes MT, et al. (2015) Inhaled nitric oxide as adjunctive therapy for severe malaria: a randomized controlled trial. Malar. J. 14:421.

27. Zheng Y, et al. (2012) In vitro microvessels for the study of angiogenesis and thrombosis. Proc. Natl. Acad. Sci. USA. 109:9342-7.

28. Min-Oo G, et al. (2003) Pyruvate kinase deficiency in mice protects against malaria. Nat. Genet. 35:357-62.

29. Ayi K, et al. (2008) Pyruvate kinase deficiency and malaria. N. Engl. J. Med. 358:1805-10.

30. Patel SN, et al. (2008) C5 deficiency and C5a or $\mathrm{C} 5 \mathrm{aR}$ blockade protects against cerebral malaria. J. Exp. Med. 205:1133-43.

31. Turner GD, et al. (1994) An immunohistochemical study of the pathology of fatal malaria. Evidence for widespread endothelial activation and a potential role for intercellular adhesion molecule-1 in cerebral sequestration. Am. J. Pathol. 145:1057-69.

32. Conroy AL, et al. (2010) Endothelium-based biomarkers are associated with cerebral malaria in Malawian children: a retrospective case-control study. PLoS One. 5:e15291.

33. Grau GE, et al. (1989) Tumor necrosis factor and disease severity in children with falciparum malaria. N. Engl. J. Med. 320:1586-91.

34. Krützfeldt J, et al. (2005) Silencing of microRNAs in vivo with "antagomirs." Nature. 438:685-9.

35. Brown H, et al. (2001) Blood-brain barrier function in cerebral malaria in Malawian children. Am. J. Trop. Med. Hyg. 64:207-13.

36. Seydel KB, et al. (2015) Brain swelling and death in children with cerebral malaria. N. Engl. J. Med. 372:1126-37.

37. Broermann A, et al. (2011) Dissociation of VE-PTP from VE-cadherin is required for leukocyte extravasation and for VEGF-induced vascular permeability in vivo. J. Exp. Med. 208:2393-401.

38. Creemers EE, Tijsen AJ, Pinto YM. (2012) Circulating MicroRNAs: Novel Biomarkers and Extracellular Communicators in Cardiovascular Disease? Circ. Res. 110:483-95.

39. Singh R, Pochampally R, Watabe K, Lu Z, Mo Y-Y. (2014) Exosome-mediated transfer of miR-10b promotes cell invasion in breast cancer. Mol. Cancer. 13:256.

40. Alexander M, et al. (2015) Exosome-delivered microRNAs modulate the inflammatory response to endotoxin. Nat. Commun. 6:7321.

41. Higgins SJ, Silver KL, Liles WC, Kain KC. (2013) Investigating the Angiopoeitin-tie2 Pathway as a Therapeutic Target to Improve Survival Following Experimental Life-Threatening Plasmodium Challenge [Abstract 1015]. In: The American Journal of Tropical Medicine and Hygiene 62nd Annual Meeting Abstract Book. Washington, DC. 89:308.

42. Lovegrove FE, et al. (2009) Serum angiopoietin-1 and -2 levels discriminate cerebral malaria from uncomplicated malaria and predict clinical outcome in African children. PLoS One. 4:e4912. 
43. Pamplona A, et al. (2007) Heme oxygenase-1 and carbon monoxide suppress the pathogenesis of experimental cerebral malaria. Nat. Med. 13:703-10.

44. Walther M, et al. (2012) HMOX1 gene promoter alleles and high HO-1 levels are associated with severe malaria in Gambian children. PLoS Pathog. 8:e1002579.

45. Serghides L, et al. (2014) PPAR $\gamma$ agonists improve survival and neurocognitive outcomes in experimental cerebral malaria and induce neuroprotective pathways in human malaria. PLoS Pathog. 10:e1003980.

46. Linares M, et al. (2013) Glutathione peroxidase contributes with heme oxygenase- 1 to redox balance in mouse brain during the course of cerebral malaria. Biochim. Biophys. Acta. 1832: 2009-18.

47. Kitamuro T, et al. (2003) Bach1 Functions as a Hypoxia-inducible Repressor for the Heme Oxygenase-1 Gene in Human Cells. J. Biol. Chem. 278:9125-33.

48. Davudian S, Mansoori B, Shajari N, Mohammadi A, Baradaran B. (2016) BACH1, the master regulator gene: A novel candidate target for cancer therapy. Gene. 588:30-7.

49. Pulkkinen KH, Ylä-Herttuala S, Levonen A-L. (2011) Heme oxygenase 1 is induced by miR-155 via reduced BACH1 translation in endothelial cells. Free Radic. Biol. Med. 51:2124-31.

50. Cheng HS, Njock M-S, Khyzha N, Dang LT, Fish JE. (2014) Noncoding RNAs regulate NF-кB signaling to modulate blood vessel inflammation. Front. Genet. 5:422.

51. Baumjohann D, Ansel KM. (2013) MicroRNAmediated regulation of $\mathrm{T}$ helper cell differentiation and plasticity. Nat. Rev. Immunol. 13:666-78.

52. Gracias DT, et al. (2013) The microRNA miR-155 controls CD8(+) T cell responses by regulating interferon signaling. Nat. Immunol. 14:593-602.

53. Seddiki N, Brezar V, Ruffin N, Lévy Y, Swaminathan S. (2014) Role of miR-155 in the regulation of lymphocyte immune function and disease. Immunology. 142:32-8.

54. Howland SW, Claser C, Poh CM, Gun SY, Rénia L. (2015) Pathogenic CD8+ T cells in experimental cerebral malaria. Semin. Immunopathol. 37:221-31.

55. Poh CM, Howland SW, Grotenbreg GM, Rénia L. (2014) Damage to the blood-brain barrier during experimental cerebral malaria results from synergistic effects of CD8+ T cells with different specificities. Infect. Immun. 82:4854-64.

56. Guermonprez P, et al. (2013) Inflammatory Flt31 is essential to mobilize dendritic cells and for $\mathrm{T}$ cell responses during Plasmodium infection. Nat. Med. 19:730-8.

57. Gordon EB, et al. (2015) Inhibiting the mammalian target of rapamycin blocks the development of experimental cerebral malaria. MBio. 6:e00725.

58. Conroy AL, et al. (2012) Angiopoietin-2 levels are associated with retinopathy and predict mortality in Malawian children with cerebral malaria. Crit. Care Med. 40:952-9.
Cite this article as: Barker KR, et al. (2017) miR-155 modifies inflammation, endothelial activation and blood-brain barrier dysfunction in cerebral Malaria. Mol. Med. 23:24-33. 